\title{
Collecting Data from a Vulnerable Population during the COVID-19 Pandemic
}

\author{
Sela R. Harcey ${ }^{1}$ \\ University of Nebraska-Lincoln \\ sharcey@,huskers.unl.edu \\ G. Robin Gauthier \\ University of Nebraska-Lincoln \\ grgauthier@unl.edu \\ Kelly L. Markowski \\ University of Nebraska-Lincoln \\ kmarkowski2@unl.edu \\ Jeffrey A. Smith \\ University of Nebraska-Lincoln \\ jsmith@unl.edu
}

Acknowledgements: This work was supported by the National Institute of General Medical Sciences of the National Institutes of Health [P20GM130461] and the Rural Drug Addiction Research Center at the University of Nebraska-Lincoln. The content is solely the responsibility of the authors and does not necessarily represent the official views of the National Institutes of Health or the University of Nebraska-Lincoln.

\footnotetext{
${ }^{1}$ Corresponding Author: Sela R. Harcey, Department of Sociology, University of Nebraska-Lincoln, 711
}

Oldfather Hall, Lincoln, NE 68555, 720-233-8497, sharcey@huskers.unl.edu 


\title{
Collecting Data from a Vulnerable Population during the COVID-19 Pandemic
}

\begin{abstract}
Conducting field research with a vulnerable population is difficult under the most auspicious conditions, and these difficulties only increase during a pandemic. Here, we describe the practical challenges and ethical considerations surrounding a recent data collection effort with a high-risk population during the COVID-19 pandemic. We detail our strategies related to research design, site selection, and ethical review. (58 words)
\end{abstract}

\section{Introduction}

Conducting field research during a pandemic raises unique practical and ethical problems for researchers (Will, Becker, and Weigand 2020). Working around a highly communicable disease requires researchers to develop new and modified protocols that mitigate the risk of contagion for both research staff and participants (de Moraes Silva, Diego R. and Mont'Alverne 2020; Dunlop et al. 2020; Gummer et al. 2020; Sastry, McGonagle, and Fomby 2020; Will et al. 2020). These problems are only amplified in research with vulnerable populations (Kohler 2020; Scherpenzeel et al. 2020). People who use drugs (PWUD), for example, are at higher risk for health problems (Bell et al. 2001; Degenhardt et al. 2017; Dombrowski et al. 2016; Lineberry and Bostwick 2006) and may be particularly vulnerable to COVID-19 (Jenkins et al. 2020; Walters et al. 2020). PWUD and other vulnerable populations may also be less willing to trust and participate in research studies, making data collection even more challenging (Costenbader, Astone, and Latkin 2006; Dunlop et al. 2020). 
We report on study protocols that were developed pre-COVID-19, which we modified for safe implementation during the pandemic. Our modified data collection efforts resulted in a sample of 28 PWUD. In what follows, we describe the strategies we employed to mitigate risks to all parties involved. We pay close attention to the balance between data quality and infection risk. We end by discussing remaining challenges and open questions for future research.

\section{Original Research Design and COVID-19 Modifications}

Our primary task was to modify the original study design to mitigate infection risk. Our original research design was developed to examine the role of drug use in daily interactions, activities, and the wellbeing of PWUD. The study included both in-person and digital components spanning four weeks. Participants would arrive at the site, complete an electronic intake survey and receive a smartphone device (Capon 2016; Roth et al. 2017; Tyler and Schmitz 2017). Using the device, participants then answer daily questions about drug use and social interactions through an app, returning to receive compensation for their responses weekly (see Appendix A). After four weeks, participants would return to complete an outtake survey, a semistructured exit interview, and collect final compensation. The intake, outtake, and weekly compensation appointments were to be held in-person in a private interview room housed at the university.

COVID-19 spreads most easily in poorly ventilated, indoor locations (CDC 2020; WHO 2020). Our original study design relied on a high frequency of in-person interactions within a single indoor space. We considered several modification options to balance data quality, participant engagement, field staff resources, and COVID-19 risk. Ultimately, we made three 
significant changes to our protocols: we moved our site outdoors, shortened the duration of the study, and shifted many interactions to a virtual format.

\section{Research Site Relocation}

The first major change we made was to move our data collection site from an indoor space to an outdoor site. Moving to an outdoor location opened up complicated questions about control over physical space, and a researcher must be prepared to negotiate with different institutional bodies to acquire the rights to a research site. Moving outside also created several unique challenges that concerned participant privacy, protocol logistics, and feasibility.

First, any study in a public outdoor setting faces the challenge of maintaining participant privacy. This is particularly true for our study population of PWUD. We did not want to 'out' any of our participants, nor did we want to expose them to other unforeseen risks (e.g., interactions with law enforcement) (Chang, Agliata, and Guarinieri 2020; Jenkins et al. 2020). Given these concerns, we chose an isolated study location. We purchased a four-sided canvas tent, where participants could complete the intake/outtake surveys. We also provided participants with headphones to use for any extended interactions that we conducted virtually (detailed more below).

Moving to an outside location created other logistical difficulties. Our protocol required that we transport study materials to and from the site and that we set up and tear down the tent daily. These requirements added at least an hour to the day. As a result, we were left with less time for in-person visits and had to schedule appointments strategically to maximize efficiency and minimize labor. 
Weather and seasonal change also was a challenge for moving our site outdoors. The pandemic delayed our ability to begin data collection, pushing the start of the study to mid-Fall, 2020. This put strong constraints on how long enrollment could continue, as potentially inclement weather in the coming months posed a threat to study completion.

\section{Reduction of Study Duration}

The second major modification of our study was the reduction of the study period. We decided to collect daily ecological momentary assessment (EMA) (Shiffman 2009; Shiffman, Stone, and Hufford 2008) data on the smartphone for 2 weeks instead of 4 weeks in order to minimize in-person interactions between staff and participants. The reduction in the EMA collection period eliminated the need for weekly compensation appointments, reducing the total in-person interactions from 5 to 2 per participant. This decision also minimized the time we were required to be outdoors (see Appendix A). The clear cost to reducing the study period was that the volume of smartphone data collected was cut in half, but we saw this as a reasonable tradeoff to reduce transmission risk for everyone involved (CDC 2020; WHO 2020).

\section{Modifying Interactions with Participants}

The last major modification to our study involved shifts in in-person interactions between ourselves and participants. This involved two important changes. First, all in-person interactions during the intake and outtake processes were modified to adhere to institutional and city-wide restrictions (Bach et al. 2020; CDC 2020). Research staff self-evaluated prior to each day of data collection and we were tested regularly for COVID-19 during the study period. We wore face coverings at all times, only removing our masks when we were outdoors, to eat or drink. We 
asked participants if they were feeling well and observed them for obvious signs of illness. Participants were required to wear masks at all times, and we offered masks to participants who arrived without them. We also attempted to maintain 6 feet of distance between ourselves and participants, although this was not always strictly possible. After each participant had completed their visits, we carefully disinfected all shared surfaces and discarded any study items (like pens), that the participant had left behind.

Our second change transitioned as many in-person interactions as possible to a virtual

format. We shifted the longer parts of the intake and outtake appointments to be conducted with a team member via Zoom. Similarly, we used a study phone to keep participants engaged throughout the study period, rather than encourage in-person drops-ins at the tent (Baruch and Holtom 2008). The study phone was manned by a single team member who spent many hours "off the clock" answering phone calls and text messages from participants. We did not lose any of our participants throughout the study period, suggesting this was a successful practice.

\section{Conclusions and Other Ethical Considerations}

This brief discussed a study of PWUD conducted during the COVID-19 pandemic. We made three main changes to our study to minimize COVID-19 risk: we relocated our site to an outdoor location; we shortened the study period; and we digitized as many interactions as possible. Our experience suggests that data can be successfully collected in-person during a pandemic, but there are unavoidable risks, stresses, and trade-offs.

In our case, the ethical question of whether or not the study could be carried out with sufficient safety was negotiated at the institutional-level and interpersonally within our research team. Our home institution required us to demonstrate strict adherence to the university's official 
COVID-19 mitigation policy and our home department required an even more stringent demonstration of precautionary measures (see Appendix B and C). Moving forward was a difficult decision because the least risky decision would have been to postpone the study indefinitely. We would not have moved forward if the entire team did not feel comfortable with the safety protocols put in place.

Ultimately, no team members tested positive for COVID-19 during the study period. Additionally, no participants experienced symptoms or left the study due to COVID-19 complications. We consider our protocols successful, as we were able to minimize risk for participants and staff while still being able to collect meaningful data.

Conducting a study with participants during a pandemic is inherently risky. Before carrying out any studies, prospective researchers must be prepared to answer several uncomfortable questions. What if the protocols do fail and a researcher or a participant falls ill? If a researcher is sick and has to quarantine (along with others who were in close contact) until they have obtained test results, will there be enough researchers to continue? If a researcher is confirmed to have contracted COVID-19, will the study be terminated? These are not the kinds of scenarios a researcher wants to anticipate, but they represent the right frame of mind when collecting data on a vulnerable population during a pandemic. 


\section{References}

Bach, Paxton, Samantha Robinson, Christy Sutherland, and Rupinder Brar. 2020. "Innovative Strategies to Support Physical Distancing among Individuals with Active Addiction." The Lancet Psychiatry 7(9):731-33. doi: 10.1016/S2215-0366(20)30231-5.

Baruch, Yehuda, and Brooks C. Holtom. 2008. "Survey Response Rate Levels and Trends in Organizational Research." Human Relations 61(8):1139-60. doi: 10.1177/0018726708094863.

Bell, David C., Doohee Lee, Su-Jau Yang, and Victoria Health. 2001. "Relationships and Diseases among Drug Users and Nonusers." Journal of Urban Health : Bulletin of the New York Academy of Medicine 78(2):313-26. doi: 10.1007/BF02410362.

Capon, Hannah. 2016. "Realising the Technological Promise of Smartphones in Addiction Research and Treatment: An Ethical Review." International Journal of Drug Policy 11.

CDC. 2020. "Coronavirus Disease 2019 (COVID-19)." Centers for Disease Control and Prevention. Retrieved November 10, 2020 (https://www.cdc.gov/coronavirus/2019ncov/more/scientific-brief-sars-cov-2.html).

Chang, Judy, Jake Agliata, and Mauro Guarinieri. 2020. "COVID-19 - Enacting a 'New Normal' for People Who Use Drugs.” International Journal of Drug Policy 83:102832. doi: 10.1016/j.drugpo.2020.102832.

Costenbader, Elizabeth C., Nan M. Astone, and Carl A. Latkin. 2006. "The Dynamics of Injection Drug Users' Personal Networks and HIV Risk Behaviors.” Addiction 101(7):1003-13.

de Moraes Silva, Diego R., and Camila Mont'Alverne. 2020. "Identifying Impacts of COVID-19 Pandemic on Vulnerable Populations." Survey Research Methods 14(2). doi: 10.18148/srm/2020.v14i2.7742.

Degenhardt, Louisa, Amy Peacock, Samantha Colledge, Janni Leung, Jason Grebely, Peter Vickerman, Jack Stone, Evan B. Cunningham, Adam Trickey, and Kostyantyn Dumchev. 2017. "Global Prevalence of Injecting Drug Use and Sociodemographic Characteristics and Prevalence of HIV, HBV, and HCV in People Who Inject Drugs: A Multistage Systematic Review." The Lancet Global Health 5(12):e1192-1207.

Dombrowski, Kirk, Devan Crawford, Bilal Khan, and Kimberly Tyler. 2016. "Current Rural Drug Use in the US Midwest." Journal of Drug Abuse 2(3).

Dunlop, Adrian, Buddhima Lokuge, Debbie Masters, Marcia Sequeira, Peter Saul, Grace Dunlop, John Ryan, Michelle Hall, Nadine Ezard, and Paul Haber. 2020. "Challenges in Maintaining Treatment Services for People Who Use Drugs during the COVID-19 Pandemic." Harm Reduction Journal 17:1-7. 
Gummer, Tobias, Claudia Schmiedeberg, Martin Bujard, Pablo Christmann, Karsten Hank, Tanja Kunz, Detlev Lück, and Franz J. Neyer. 2020. "The Impact of Covid-19 on Fieldwork Efforts and Planning in Pairfam and FReDA-GGS." Survey Research Methods 14(2). doi: $10.18148 / \mathrm{srm} / 2020 . v 14 i 2.7740$.

Jenkins, Wiley D., Rebecca Bolinski, John Bresett, Brent Van Ham, Scott Fletcher, Suzan Walters, Samuel R. Friedman, Jerel M. Ezell, Mai Pho, John Schneider, and Larry Ouellet. 2020. "COVID-19 During the Opioid Epidemic - Exacerbation of Stigma and Vulnerabilities.” The Journal of Rural Health. doi: https://doi.org/10.1111/jrh.12442.

Kohler, Ulrich. 2020. "Survey Research Methods during the COVID-19 Crisis." Survey Research Methods 14(2). doi: 10.18148/srm/2020.v14i2.7769.

Lineberry, Timothy W., and J. Michael Bostwick. 2006. "Methamphetamine Abuse: A Perfect Storm of Complications." Mayo Clinic Proceedings 81(1):77-84. doi: 10.4065/81.1.77.

Roth, Alexis M., Marisa Felsher, Megan Reed, Jesse L. Goldshear, Quan Truong, Richard S. Garfein, and Janie Simmons. 2017. "Potential Benefits of Using Ecological Momentary Assessment to Study High-Risk Polydrug Use." MHealth 3:46-46. doi: 10.21037/mhealth.2017.10.01.

Sastry, Narayan, Katherine McGonagle, and Paula Fomby. 2020. "Effects of the COVID-19 Crisis on Survey Fieldwork: Experience and Lessons From Two Major Supplements to the U.S. Panel Study of Income Dynamics." Survey Research Methods 14(2). doi: 10.18148/srm/2020.v14i2.7752.

Scherpenzeel, Annette, Kathrin Axt, Michael Bergmann, Salima Douhou, Andrea Oepen, Gregor Sand, Karin Schuller, Stephanie Stuck, Melanie Wagner, and Axel Börsch-Supan. 2020. "Collecting Survey Data among the 50+ Population during the COVID-19 Outbreak: The Survey of Health, Ageing and Retirement in Europe (SHARE)." Survey Research Methods 14(2). doi: 10.18148/srm/2020.v14i2.7738.

Shiffman, Saul. 2009. "Ecological Momentary Assessment (EMA) in Studies of Substance Use." Psychological Assessment 21(4):486-97. doi: 10.1037/a0017074.

Shiffman, Saul, Arthur A. Stone, and Michael R. Hufford. 2008. "Ecological Momentary Assessment." Annual Review of Clinical Psychology 4(1):1-32. doi: 10.1146/annurev.clinpsy.3.022806.091415.

Tyler, Kimberly A., and Rachel M. Schmitz. 2017. "Using Cell Phones for Data Collection: Benefits, Outcomes, and Intervention Possibilities with Homeless Youth." Children and Youth Services Review 76:59-64. doi: 10.1016/j.childyouth.2017.02.031.

Walters, Suzan M., David W. Seal, Thomas J. Stopka, Megan E. Murphy, and Wiley D. Jenkins. 2020. "COVID-19 and People Who Use Drugs - A Commentary." Health Behavior and Policy Review 7(5):489-97. doi: 10.14485/HBPR.7.5.11. 
WHO. 2020. "Transmission of SARS-CoV-2: Implications for Infection Prevention Precautions." World Health Organization. Retrieved November 10, 2020 (https://www.who.int/news-room/commentaries/detail/transmission-of-sars-cov-2implications-for-infection-prevention-precautions).

Will, Gisela, Regina Becker, and Dominik Weigand. 2020. "COVID-19 Lockdown during Field Work." Survey Research Methods 14(2). doi: 10.18148/srm/2020.v14i2.7753. 


\section{Appendix A.}

Appendix A. Breakdown of Modified Study Procedures for COVID-19 Implementation

\begin{tabular}{|c|c|c|}
\hline & $\begin{array}{l}\text { Pre-COVID-19 } \\
\text { Procedures }\end{array}$ & $\begin{array}{l}\text { COVID-19 Modified } \\
\text { Procedures }\end{array}$ \\
\hline $\begin{array}{l}\text { Points of Contact } \\
\text { 1. Intake Appointment } \\
\text { a. Consent Process } \\
\text { b. Intake Survey } \\
\text { 2. Week } 1 \text { Compensation Appointment } \\
\text { 3. Week } 2 \text { Compensation Appointment } \\
\text { 4. Week } 3 \text { Compensation Appointment } \\
\text { 5. Outtake Appointment } \\
\text { a. Outtake Survey } \\
\text { b. Exit Interview }\end{array}$ & $\begin{array}{l}\text { All in-person } \\
\qquad \begin{array}{r}\mathrm{X} \\
\mathrm{X} \\
\mathrm{X} \\
\mathrm{X} \\
\mathrm{X} \\
\mathrm{X} \\
\mathrm{X}\end{array}\end{array}$ & $\begin{array}{l}\text { All in-person (modified) } \\
\qquad \begin{array}{l}\text { X (Virtual) } \\
\text { X } \\
- \\
- \\
- \\
\text { X } \\
\text { X (Virtual) }\end{array}\end{array}$ \\
\hline Total In-Person Interactions & 5 & 2 \\
\hline Study Duration & 4 weeks & 2 weeks \\
\hline Research Site & Indoor Interview Room & Outdoor Canvas Tent \\
\hline
\end{tabular}


Appendix B. Forms Required to Restart Human Subjects Research

\section{Restarting In-Person Human Subjects Research Plan to restart in-person human subjects research (online form: https://research.unl.edu/events/event2.php?event|D=4795)}

Principle Investigator Name and E-mail:

First Name:

Last Name:

Email Address:

Department, School or Unit:

Chair/Head/Director Name:

Center of Institute (if applicable):

Provide a brief description up to four sentences about the nature of your study or studies to give context for how your research team interacts with research participants.

If you will utilize a core facility, such as an imaging facility, please contact the core facility's director to request a copy of its reopening plan that will have identified safety measures being taken in the facility. You may reference that reopening plan rather than restating its contents when responding to the remainder of this form.

Core Facility Name (if applicable):

\section{Personal Hygiene, Prevention and Well-being Measures}

You are expected to comply with the personal hygiene, prevention and well-being measures outlined in the Forward to Fall Guiding Framework. It is especially important to communicate to research participants the expectation that they also follow these measures both while on-campus and while interacting with the research team. You may choose to utilize the COVID-19 Information Sheet for Research Participants to assist in communicating with potential in-person human subjects research participants. If you are unable to comply with these safety measures, you must have an approved exemption in place prior to requesting departmental approval to conduct in-person human subjects research. Exemption approvals should be attached to this plan as additional supporting correspondence.

Detail the communication related to COVID-19 that will occur with research participants prior to and during the visit. 
Detail the plans for UNL faculty, staff, students, and research participants to utilize personal protective equipment (PPE) when interacting with one another.

Confirm adequate supply of lab attire and PPE (face coverings, gloves, etc.). If you need to obtain additional lab attire or PPE and are unable or unsure how to obtain the quantities you need, please work with your unit leader or associate dean for research to identify the best way to obtain the items. The campus has ordered large quantities of disposable masks and other items in anticipation of restarting in-person human subjects research. Those PPE items are on campus and can be requested here.

Do you have adequate Supply of lab attire and PPE?

Yes

No

If you are unable to comply with the personal hygiene, prevention and well-being measures outlined in the Forward to Fall Guiding Framework, such as your research team or research participants being unable to wear an approved face covering, what approved actions will you take to ensure the safety of the research team and the research participant?

Additional precautionary actions that will be taken:

\section{Public Hygiene}

Plans should be developed to ensure there is readily available and easy access to hand sanitizing products as well as disinfecting supplies for high-touch points and areas with limited service by custodial staff.

Ensure adequate supply of hand sanitizer and disinfecting supplies.

Ensure cleaning/disinfecting between research participants/visits and adequate cleaning/disinfecting supplies, testing equipment, etc., before and after use by each participant.

\section{Facilities, Physical Distancing and Density Reduction}

All university activities, wherever performed, are expected to maintain physical distancing requirements of 6 feet between participants unless an exemption has been approved in advance. Exemption approvals should be attached to this plan as additional supporting correspondence.

Will interactions with research participants occur on the UNL campus or off? 
$\square$ On

Off

If off campus, what are the location(s) where interactions will occur?

If off campus, follow UNL guidelines plus guidelines of location you are visiting. If conflicting, you must follow the more stringent requirements.

If on campus, have you also completed a Research and Creative Space Reopening Plan and Checklist?

Yes

No

If you are unable to comply with the physical distancing requirements outlined in the Forward to Fall Guiding Framework, what approved actions will you take to ensure the safety of the research team and the research participant (e.g., use of Plexiglass shield)?

Additional precautionary actions that will be taken:

\section{Travel}

Does the in-person human subjects research require members of your research team (faculty, staff, or student workers) to travel?

Yes

No

If yes, are you prepared to comply with the current travel guidelines, including restricting the number of individuals in a vehicle?

$\square$ Yes

$\square$ No

Current travel guidelines.

\section{IRB Approval}

If your funding agency or sponsor requires you to obtain additional approvals from the IRB before reinitiating your research in the COVID-19 pandemic context, please review the IRB FAQs and notify the IRB that you require this additional approval. 
Appendix C. Required Reopening Plan and Checklist

\section{Research and Creative Space Reopening Plan and Checklist (online form: https://research.unl.edu/events/event2.php?eventID=4688)}

UNL researchers who plan or need to reopen campus research and creative spaces (e.g., laboratories, studios) to resume on-campus activities first must provide a plan to demonstrate their activities can be done safely and with appropriate protection against the spread of the SARS-CoV-2 virus. Faculty must submit the following plan and checklist for approval by their department chair/head/director; college dean or dean's designee; and the vice chancellor for research and economic development or the VCRED's designee.

Principal investigators should consult the Guidelines for Opening UNL Research Labs when preparing their reopening plan.

These plans will take effect June 1 and are expected to be in place until the university issues further guidance, either implementing a less or more restrictive research work environment. We expect that change to occur no sooner than July 1, which is when the current Lincoln-Lancaster County Health Department Directed Health Measures are scheduled to expire.

\section{Reopening Plan}

Principal Investigator Name:

First Name:

Last Name:

Email Address:

Department, College and/or Center/Institute:

Building name and room(s):

Names of all faculty, postdocs, staff, and students working on-site in the research space. Please indicate estimated on-campus schedules, days/times, where applicable.

Describe planned physical work arrangements for labs and research spaces (one person per bay, alternating benches, etc.)

Identify de-densification plans such as alternating days or extended hours to minimize the number of people on-site at one time and to enable social distancing. 
Describe plans to clean and disinfect laboratories and any workspaces (include frequency and type of cleaner and disinfectant)

List any core facilities (animal facility, imaging, omics etc.) you will utilize and detail what work must occur in university spaces that are outside your research space

Detail the proposed laboratory attire and personal protective equipment (PPE) that will be used by personnel, including facial coverings or respiratory protective equipment. If this varies by task, describe.

Confirm adequate supply of lab attire and PPE (lab coats, gloves, gowns, eyewear, face coverings, etc.).

If you need to obtain additional lab attire or PPE are unable or unsure how to obtain the quantities you need, please work with your unit leader or associate dean for research to identify the best way to obtain the items. Limited quantities of some PPE are available for purchase on campus and can be requested here.

Upload additional supporting correspondence or documentation.

Attachments:

\section{Reopening Checklist}

All faculty, postdocs, staff and students should complete training and review supplemental guidance on the following prior to returning to on-site work: 
- Training on COVID-19 Awareness - Campus Procedures \& Self-Care

- Expected use and care of facial coverings (Centers for Disease Control guide).

- Social distancing practices recommended by the CDC.

- Enroll in UNL's Respiratory Protection Program or Hearing Conservation Program if intending to use NIOSH-approved respirators or hearing protectors. Consult UNL Environmental Health and Safety if intending to use any type of respiratory protection other than cloth facial coverings or surgical-like masks.

Are personnel reporting to campus only when their physical presence is required? Ensure you have the following available:

- Adequate supply of supplies for hand washing and hand sanitizing?

- Adequate supply of Environmental Protection Agency-approved cleaning and disinfecting supplies to clean surfaces frequently?

- Access to appropriate disposable and/or cloth face coverings?

Review the EHS safe operating procedure (SOP) on Laboratory Commissioning, particularly the section regarding re-opening a laboratory after a period of hibernation and implementing corrective action, as necessary.

- $\quad$ EHS safe operating procedure (SOP) on Laboratory Commissioning

Review the EHS SOP on Laboratory Cleaning and Disinfection. Consider identifying high-touch areas in the space with a schedule for disinfecting these areas on a more frequent basis.

- EHS SOP on Laboratory Cleaning and Disinfection

Has applicable safety and hygiene signage been posted in the space?

- Download COVID-19 signage

Have you reviewed De-Densification Occupancy Guidance and requested physical modifications and/or made any workstation labels and floor markings needed to provide a safe working space?

- De-Densification Occupancy Guidance

- Request physical modifications

- Workstation labels and floor markings guidance

Examples include plexiglass shields/dividers, removing chairs and adding floor labels.

Is this a new plan or a revision?

New plan

Revision to previous plan 